\title{
Selective impairment of decision making under ambiguity in alexithymia
}

\author{
Lei Zhang ${ }^{1,3,4}$, Xue Wang ${ }^{1,3}$, Yu Zhu ${ }^{1,3}$, Hongchen $\mathrm{Li}^{1,3}$, Chunyan Zhu ${ }^{1,3,4}$, Fengqiong Yu ${ }^{1,3,4}$ and Kai Wang ${ }^{2,1,3,4^{*}}$
}

\begin{abstract}
Background: Alexithymia is characterised by difficulties identifying and describing emotions. Few studies have investigated how alexithymia influences decision-making under different conditions (ambiguity and risk). This study aimed to examine whether alexithymia contributes to impairment in decision-making.

Method: This study included 42 participants with high scores in the Chinese version of Toronto Alexithymia Scale (alexithymia group), and 44 matched subjects with low scores (control group). Decision-making was measured using the lowa Gambling Task (IGT) and the Game of Dice Task (GDT).

Results: The main findings of this study revealed selective deficits in IGT performance for the alexithymia group, while GDT performance was unimpaired when compared with the control group. In IGT, total netscores were lower for the alexithymia group compared to the control group, particularly with regard to block 5. Moreover, the alexithymia individuals selected significantly more adverse cards than the controls, indicating significant decisionmaking impairments.
\end{abstract}

Conclusion: Alexithymia selectively influences decision-making under ambiguity.

Keywords: Alexithymia, Decision making, TAS-20, Ambiguity, Risk

\section{Background}

Several recent studies have supported the hypothesis that emotions may modulate human behaviour and cognition, and many researchers have extensively examined the influence of negative emotions on higher cognitive functions [1]. From an evolutionary perspective, negative emotions, such as anxiety, serve to signal a potential threat in the environment [2]. However, inappropriate activation of the negative emotion system may contribute to the onset of some psychiatric disorders [3]. Alexithymia (ALEX) is typically characterised by difficulties describing, understanding or identifying self-other emotions. This condition was originally thought to be a syndrome exhibited by patients with psychosomatic and psychiatric disorders [4]. Currently, alexithymia is understood as a personality trait, the severity of which varies across different populations [5]. Several studies have found that

\footnotetext{
* Correspondence: wangkai1964@126.com

${ }^{2}$ Department of Neurology, the First Affiliated Hospital of Anhui Medical University, Hefei, China

${ }^{1}$ Department of Medical Psychology, Chaohu Clinical Medical College, Anhui Medical University, Hefei, China

Full list of author information is available at the end of the article
}

individuals with alexithymia demonstrate poor performance on executive and cognitive tasks associated with prefrontal cortex [6], and are related with a decline representational function regarding decision-making [7].

Decision-making is an important and complex cognitive function including assessing and evaluating the short- and long-term costs and benefits that may be attributed to different options. In the field of neuroscience, two types of decision-making differ mainly with regard to the level of unsureness concerned as well as how much useful information is offered to the operator in terms of the consequences of decisions and their associated probabilities [8]. In some cases, consequences and possibilities are uncertain. In order to determine the viability of the options available, decision-makers must assess relevant information according to feedback based on the previous selection. The form of decision-making is described 'decision-making under ambiguity' and is commonly evaluated using the Iowa Gambling Task (IGT) [9]. In the IGT, choosing cards from advantageous decks results in maximum profit. Participants need to overcome an initial attraction to high-payoff decks with 
subsequent big punishments. Contrary to the ambiguous decision-making, for some aspects of decision, explicit information is provided concerning the possible results of various options and their associated probabilities. This is known as decision-making under risk, and is evaluated using the Game of Dice Task (GDT) [10]. In GDT, participants are asked to choose among four different choices which clearly related to winning or losing. In addition, the probabilities associated with winning are clear and stabilized before participants begin testing. Some options are related to high latency wining / losing, while other options are associated with lower potential wining / losing. In planning to win as much money as possible, participants must consider which options will lead to more benefits. Personality and emotion are important factors in risky decision making [11]. Alexithymia is a personality trait, typically characterized by inability to understand, describe and explain one's emotions. In addition, there is evidence suggesting that the reduced ability to make emotional appraisals in alexithymia affects the performance of risk decision tasks $[12,13]$. Therefore, we speculated that alexithymia could modulate risky decision making.

Neuroimaging and neuropsychological studies have identified several brain areas that are correlated with decision-making, including the ventromedial prefrontal cortex (vmPFC), the dorsolateral prefrontal cortex (dlPFC), the orbitofrontal prefrontal cortex (OFC) and the anterior cingulate cortex (ACC) [14-16]. Deficient IGT performance, for example, the preference to select adverse decks, was presented in patients with vmPFC / OFC lesions [16, 17]. Patients with vmPFC lesions exhibit a significantly greater reflection effect, choosing more gambles under the condition of losing and fewer gambles under the condition of wining [17]. Depressive patients with suicide history that were related with dysfunction of left lateral orbitofrontal cortex showed alterations in the processing of risk under conditions of uncertainty [16]. However, some articles have shown that the dlPFC has an important influence in performance on the GDT. Patients with dlPFC dysfunction showed impairment of performance on the GDT $[18,19]$. Moreover, risky decision-making evaluated by the GDT, rests on activation of the dIPFC [20]. While these brain areas are associated with decision-making, they also have a significant impact on alexithymia. Several neuroimaging researches have suggested that alexithymia is associated with decreased activation of the vmPFC [21], reduced orbitofrontal cortical thickness [22] and altered cognition-related brain activity within the dIPFC [23]. Thus, alexithymia may influence the two different patterns of decision making.

Few studies have investigated decision-making under different conditions (ambiguity and risk) in alexithymia.
As decision-making involves the cognitive capacity to process emotions [24, 25], this current study used the IGT and the GDT to examine whether alexithymia contributes to deficits in decision-making. We hypothesized that alexithymia would have deficits in their decision-making function. Moreover, we compared the different performances between the two decision making tasks in alexithymia. As GDT performance may be determined by some certain executive functions, such as monitoring and classification, and use of feedback without persistent tendency (as measured by the Wisconsin Card Sorting Test (WCST)) [26, 27], and in order to balance the differences in executive functions related to performance of GDT, subjects completed WCST [28].

\section{Methods}

\section{Participants}

Five hundred forty students who were in Grade Two and from Anhui Medical University were assessed for alexithymia using the Chinese version of the Toronto Alexithymia Scale (TAS-20) [29]. Finally, 513 valid questionnaires were returned. The sample included 284 male and 229 female with an average age of 19 . The average score of the TAS20 of 513 students was approximately 49.2. We selected the cut-off for high alexithymia - ALEX group - >60 reflecting the top quartile score; and cut-off for low alexithymia - Control group $-<40$ reflecting the bottom quartile score, in order to obtain a sample with as large a variance in alexithymia as possible [30]. All participants satisfied the following exclusion criteria: (a) no history of substance abuse (including alcohol), (b) no demonstrable brain disorder (e.g., epilepsy, schizophrenia, brain injury or head trauma), (c) no depressive or anxiety disorder (i.e., exclusion of participants who scored $\geq 41$ as measured using the Self-Rating Depression Scale [SDS]; exclusion of participants who scored $\geq 40$ as measured using the SelfRating Anxiety Scale [SAS]).

Finally, the selected sample consisted of 42 students scored higher than 60 in the alexithymia group (mean = 64.31; $\mathrm{SD}=2.63$ ) and 44 students scored lower than 40 in the control group (mean $=36.82 ; \mathrm{SD}=2.34$ ). Participants ranged from 20 to 21 years of age. In these two groups, the ratio of female to male was similar. (Table 1). This study was approved by the ethics committee at Anhui Medical University. Written informed consents were obtained from all the participants and $¥ 20$ was accepted for their participation.

\section{Procedure}

\section{SDS and SAS}

SDS is a self-reported 20-item questionnaire that generally considered a reliable instrument for measuring depressive symptoms in the general population [31]. 
Table 1 Demographic data of alexithymia and control groups

\begin{tabular}{lllll}
\hline & $\begin{array}{l}\text { Alexithymics } \\
(n=42)\end{array}$ & $\begin{array}{l}\text { Controls } \\
(n=44)\end{array}$ & $\mathrm{t} / \mathrm{x} 2$ & $P$ \\
\hline Age (years) & $19.23(0.48)$ & $19.25(0.44)$ & 0.12 & 0.91 \\
Gender (male/female) & $21 \mathrm{~m} / 21 \mathrm{f}$ & $23 \mathrm{~m} / 21 \mathrm{f}$ & 0.04 & 0.83 \\
Education (years) & $14.19(0.40)$ & $14.25(0.44)$ & 0.66 & 0.51 \\
SDS & $34.23(5.77)$ & $32.07(6.29)$ & 1.22 & 0.23 \\
SAS & $33.18(4.74)$ & $30.78(5.57)$ & 1.36 & 0.17 \\
TAS-20 (score) & $64.31(2.63)$ & $36.82(2.24)^{* * *}$ & 52.3 & 0.000 \\
WCST & & & & \\
wrong responses & $19.79(8.81)$ & $18.23(6.37)$ & 0.93 & 0.35 \\
perseverative response & $38.38(7.37)$ & $37.86(6.21)$ & 0.35 & 0.73 \\
perseverative errors & $12.78(5.32)$ & $12.69(6.42)$ & 0.07 & 0.95 \\
\hline${ }^{*} p<.05, * * 0.01$ and ${ }^{* * *} p<.001$ & & &
\end{tabular}

Each item is this questionaire is scored from 1 (rarely) to 4 (frequently), according to the frequency that symptoms affect the subjects' life. SAS is a 20-item measure developed to assess the frequency of anxiety symptoms [32]. The four-point scoring method was applied in this questionnaire (from 1 point to 4 points), according to severity of symptoms. A higher score indicates more serious anxiety symptoms.

\section{Decision-making under ambiguity}

The computerised version of the Iowa Gambling Task is a usual method of measuring decision making under condition of uncertainty [33]. The IGT assesses the ability to choose between outcomes that yield high incomes with the high risk of future loss, low incomes with small risk of loss. In this task, subjects were asked to accumulate as much money as possible by selecting one card from each of the four decks (A, B, C and D) at a time, until 100 cards were chosen. Following each card selection, the message "You get 100" or "You get 100 but lose 200" appeared on the screen immediately. Decks A and $\mathrm{B}$ were disadvantageous decks. These decks yielded high instant returns and higher losses, leading to long-term negative consequences. In contrast, decks $\mathrm{C}$ and $\mathrm{D}$ were beneficial. These decks yielded small instant gains, but the losses are small, resulting in long-term positive consequences. In addition, there were additional differences between the four decks. Although both A and B decks were unfavorable decks, the choices of the deck A were penalized in $50 \%$ of the trials, whereas deck B choices were penalized in $10 \%$ of the trials. Similar differences were evident with regard to decks C (50\% losses) and D (10\% losses). The instant losses for deck D were larger than those for deck $C$ [34]. The netscore was measured by calculating the scores associated with card selections from advantageous decks $(\mathrm{C}+\mathrm{D})$ and disadvantageous decks $(A+B)$. One hundred choices were equally divided into 5 blocks. Calculation of netscore for each block was assessed changes in decision making over time. Typically, the netscore, $(C+D)-(A+B)$, is used to analyse the results obtained from the IGT.

\section{Decision-making under risk}

In the computerised GDT, a virtual shaker and a single die appears on the screen [10]. Participants were asked to maximise their fictitious start-up funds $(€ 1000)$ within 18 dice throws by selecting one of four different options. Before each throw, participants had to choose either a single die or one die from two, three or four dice combinations. Each choice is related to particular fictitious gains and losses, based on the probability of occurrence: $€ 1000$ gain/loss for the choice of a single number with probability of winning 1:6; $€ 500$ gain/loss for a combination of double numbers with probability of winning 2:6; $€ 200$ gain/loss for a combination of triple numbers with probability of winning 3:6; and $€ 100$ gain/ loss for a combination of quadruple numbers with probability of winning 4:6. The winning probability attributable to the different selections may be deduced easily by referring to the ratio of occurrence $(1: 6,2: 6,3: 6$, and 4:6). For instance, if a participant bets on a single number "one", and one is thrown, the participant wins $€ 200$; however, if a two, three, four, five, or six is thrown, the participant loses $€ 200$. The first two choices were classified as risky decisions for lower winning probabilities; the two latter choices were classified as safe decisions for higher winning probabilities. Furthermore, after each option, some changes appeared on the screen including the number of remaining dice, gain or loss and capital change. To analyse risky decisions, the netscore was calculated (the number of safe options minus the number of risky options) to analyse task performance. In addition, the frequency of each option was analysed separately.

The order across the two tests was counterbalanced by a Latin-square design and all subjects could rest if they felt fatigued. At the end of the experiment, subjects were asked whether they were interested in the experiment and what kind of experiences they felt during the experiment. The whole experiment lasted approximately $20 \mathrm{~min}$.

\section{Statistical analysis}

Statistical analysis was carried out with SPSS version 17. A repeated measures analysis of variance (ANOVA) was used, with the trial blocks (IGT) and different types of choices (GDT) as the within-subjects factor and study group as the between-subjects factor, to examine the influence of performance on decision-making tasks under different conditions. Post hoc test was used to test the effects of the different process of decision-making in the IGT. A one-way ANOVA was performed to analyse 
the effects of four different types of choices in GDT. The statistical significance level was $p<.05$.

\section{Results}

No statistically significant differences were found in terms of age, gender, educational level, SDS or SAS score between the two groups. Notably, there was no significant difference in WCST scores between the two groups. The results illustrated that the ALEX group have a similar executive function when compared to the control group (Table 1).

\section{IGT and GDT}

To examine the IGT and GDT performances, a repeated measures ANOVA was conducted, with group as between-subjects factor and task as the within-subjects factor. There was a significant main effect for task $\left(F_{1,84}\right.$ $=23.87, p=.000)$, and for a group by task interaction $\left(F_{1,84}=8.68, p=.004\right)$. According to the post hoc LSD tests, the netscores of IGT in the two groups were significantly lower compared to the netscores of GDT ( $p$ $=.001$ ). Pair wise comparisons indicated a remarkable difference between the total IGT netscores of the two groups $\left(t_{1,84}=-2.13, p=.036\right)$ (Fig. 1a). The result

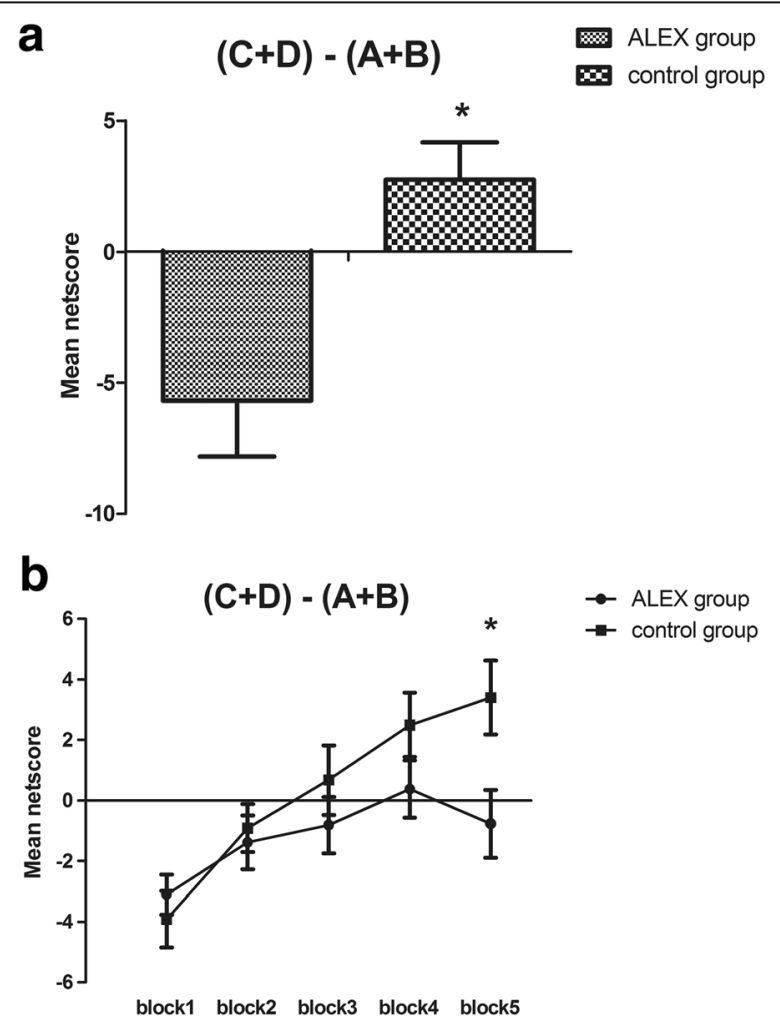

Fig. 1 Total netscore during the lowa Gambling Task (IGT) and netscore of the five blocks in the task between two groups. (C+D)-(A $+B)$ : the number of advantageous choices minus the number of disadvantageous choices ${ }^{*} p<.05,{ }^{* *} p<.01$ and ${ }^{* *} p<.001$ showed that the beneficial decks selected in the control group were more than those in the ALEX group. Contrary, the difference was not statistically significant in the netscores of the GDT between the two groups $\left(t_{1,84}\right.$ $=1.49, p=.14)$. Furthermore, in the ALEX group, the significant difference the was found between IGT and GDT netscores $\left(t_{1,41}=4.99, p=.000\right)$. However, in the control group, the difference was not statistically significant $\left(t_{1,43}=1.54, p=.13\right)$.

\section{Decision-making in the IGT Netscore in the IGT}

In order to investigate the IGT performances in detail, a repeated measure ANOVA was conducted, with group as the between-subjects factor and block as the withinsubjects factor. The main effect for group was significant $\left(F_{1,84}=4.02, p=.048\right)$, showing that performances in the control group outperformed those in the ALEX group; for block $\left(F_{4,336}=9.48, p=.000\right)$, indicating a dynamic process of change in IGT; and for a block by group interaction $\left(F_{4,336}=5.21, p=.025\right)$. In the IGT experiment, the change of decision strategy is reflected by the change curve. Post hoc LSD tests revealed that the difference was not statistically significant between adjacent blocks on the netscore of the ALEX group ( $p=.19$, $p=.66, p=.36, p=.38$ ). However, there was a significant effect of decision process between block 1 and block 2 and between block 3 and block 4 on the netscore of the control group $(p=.03, p=.046)$, showing that the decision making strategies of the control group changed significantly with the process of the task. In addition, simple effect analysis of netscores of five blocks suggested statistical difference for block 5 between the two groups $(p=.014)$ (Fig. 1b).

\section{Individual deck level preference}

In order to examine the trend of individual deck level in IGT, a repeated measure ANOVA was used. There was a significant main effect for deck $\left(F_{3,252}=5.91, p=.02\right)$, and for a group by deck interaction $\left(F_{3,252}=3.17, p=.048\right)$. Simple effect analysis of performances on the four types of decks between the two groups indicated that significant statistical difference existed in the score of deck A $(p=.044)$. The results suggested that the Alex group chose more adverse options than the control group (Fig. 2).

\section{Decision making in the GDT GDT netscores}

By comparison,no significant difference was found in the netscores of the GDT between the two groups (Fig. 3a). The collecting dates were analysed by repeated measurement analysis of variance, with group as the betweensubjects factor and option as the within-subjects factor. For 


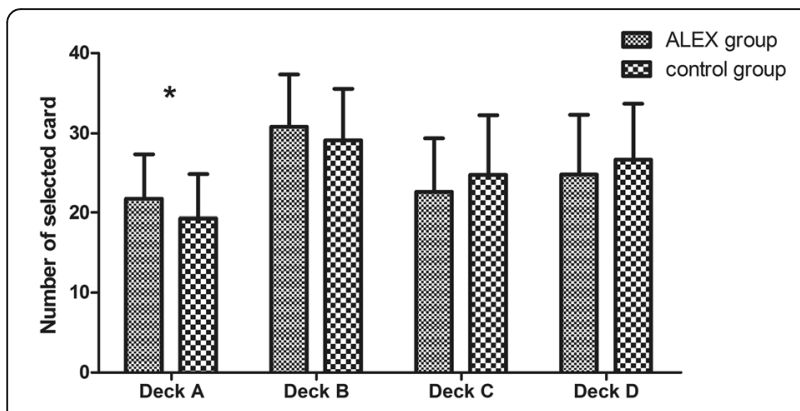

Fig. 2 Number of four types of cards selected for two groups during the lowa Gambling Task (IGT). ${ }^{*} p<.05,{ }^{* *} p<.01$ and ${ }^{* *} p<.001$

option, main effect of option was significant $\left(F_{3,252}=18.73\right.$, $p=.000)$. However, the main effect of group had no significant statistical difference $\left(F_{1,84}=.95, p=.33\right)$, and no significant option by group interaction $\left(F_{3,252}=.97, p=.42\right)$. With regard to the rate of each type of selection, none of the simple comparisons had significant differences between both groups: one single number $\left(t_{1,84}=-0.42, p=.68\right)$; two numbers together $\left(t_{1,84}=-0.84, p=.40\right)$; three numbers together $\left(t_{1,84}=1.65, p=.10\right)$; four numbers together $\left(t_{1,84}=-0.41, p\right.$ $=.69)$ (Fig. 3b).

Following the selection of a risky option, this study explored the utilisation of negative feedback (loss) in GDT to choose a safe option. Those subjects who chose a risky choice and accepted negative feedback once or more were selected. Therefore, a total data of 74 subjects

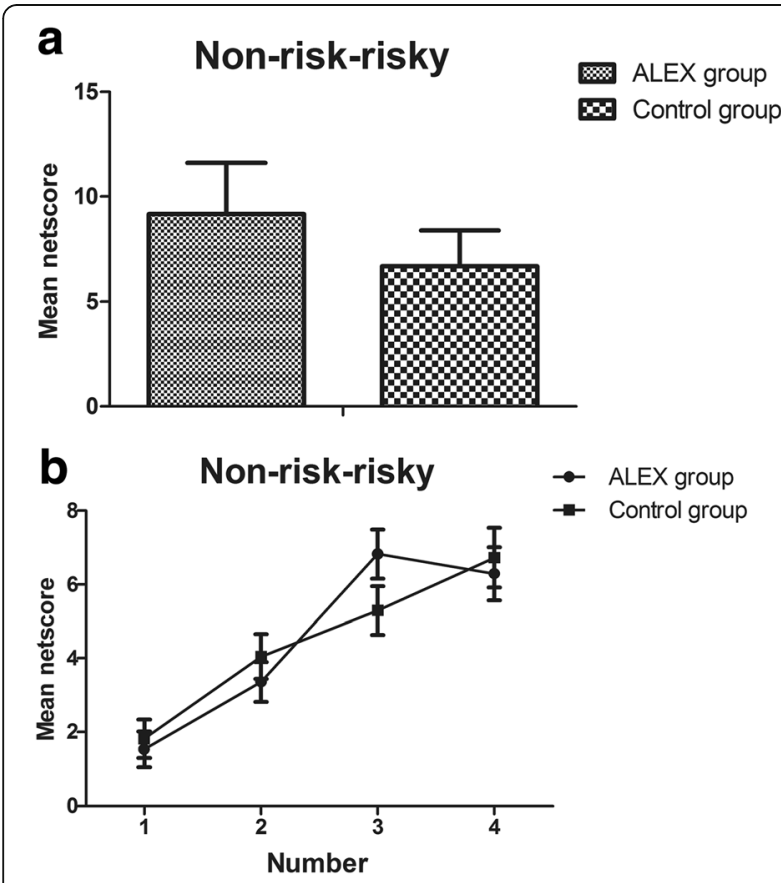

Fig. 3 Netscore and mean frequency of each alternative during the Game of Dice Task (GDT) between two groups. Non-risky-risky: the number of safe choices minus the number of risky choices were collected and analysed. Both groups had no significant difference concerning the utilisation of negative feedback $\left(t_{1,72}=1.67, p=.10\right)$. No significant correlation existed between the netscore of GDT and the utilisation of negative feedback (ALEX group: $r=0.02, p=.87$; control group: $r=0.17, p=.085)$. The utilisation of positive feedback (gains) was in those who decided on a safe choice in choosing a safe choice again in the following trial. Those subjects who selected a safe choice and accepted positive feedback once or more were selected. Therefore, a total data of 82 subjects were collected and analysed. The two groups differed significantly in the aspect of the utilisation of positive feedback $\left(t_{1,80}=1.27\right.$, $p=.21$ ). In addition, no significant correlation existed between the netscore of GDT and the utilisation of positive feedback (ALEX group: $r=0.12, p=.11$; control group: $r=0.20, p=.067$ ).

\section{Discussion}

This current study investigated two decision-making situations using participants with alexithymia and a control group. The main findings show that participants with alexithymia demonstrated selective deficits in IGT performance but unimpaired GDT performance when compared with the control group. Total IGT netscores were lower for the ALEX group than for the control group, particularly with regard to block 5. Furthermore, both two groups showed distinct trends of individual deck level for the IGT: the ALEX group chose more adverse options than the control group, indicating significant decision-making impairments.

The ALEX group performed worse than the control group in the IGT. The results suggest that participants in the ALEX group were preferred instant income and were difficult to develop an effective strategy in the long run. This result corresponds with previous findings that suggested a correlation between alexithymia and deficits in decision-making abilities, indicating that alexithymia may be a critical personality trait underlying decisionmaking deficits [7]. Poletti et al. found that total IGT scores did not differ between alexithymic and nonalexithymic patients with Parkinson's disease. However, significant differences emerged across the third block of the IGT [35]. Alexithymic gamblers chose less advantageously in this task than non-alexithymic gamblers in the IGT. The more severity of alexithymia appeared, the more severity of the deficit in decision-making abilities damaged [7]. As anxiety and depression are recognised as factors that may impair decision-making abilities [36, 37], subjects who had high levels of depression and anxiety were excluded through the SDS and the SAS. Comparable to the findings of previous research [38, 39], alexithymia appears to be a stable personality trait rather than a phenomenon [7]. In addition, it may be noted 
that total IGT scores were lower for the ALEX group than for the control group, particularly with regard to block 5 . This finding is supported by the consolidationattenuation model, in view of a mounting evidence that suggests a correlation between alexithymia and deficits of information processing concerning emotional arousal [40], as well as emotional-based memory [41]. Thus, during the process of experiment, alexithymia individuals may have declining ability of continuous learning and difficulty to use experience to guide future behaviour which would otherwise lead to attenuated learning.

Importantly, for the IGT, the findings of this current study show that the ALEX group selected more deck A cards than the control group, indicating a significant decision-making impairment. The clinical manual of IGT states that decks A and B are adverse options, and that selections of deck A is avoided by most "neurologically intact" individuals [34]. As previously outlined, selections of deck B may not discriminate effectively between the decision-making performances of subjects in both two groups, while continuous selections from deck $\mathrm{A}$ are more indicative of pathological risk-taking [34]. Thus, while deck B was sensitive to risky decisionmaking, deck A was more sensitive to decision-making impairment. Current research suggests that there may be evident correlations between personality characteristics and deck A selections among non-clinical and clinical populations, for example, individuals with trait anxiety, substance abuse/dependence and obsessivecompulsive disorder $[18,42,43]$. Our result is in accordance with the contention that alexithymia related differently to in the selection of deck A compared to controls.

In contrast to the results from the IGT, no performance differences in the GDT were found between the alexithymia group and the control group. Previous studies that examined patients with various diseases as well as normal participants showed a correlation between disadvantageous decisions in risky decision-making and low executive performance, including task switching, flexibility of cognition and categorisation as measured by the WCST [44]. A patient who underwent brain resection for glial cyst was examined using the cognitive tasks, executive function tests and intelligence test. Results showed selective deficits in decision making and executive functions, compare with other cognitive components [45]. Furthermore, additional studies found that participants with intact executive functions showed no impairment in performance in the GDT $[46,47]$. Consequently, previous research suggests that the association is most attributable to participants' utilisation of information concerning the possible options of GDT that obviously involved planning, monitoring and modification of favorable strategies. In summary, we hypothesised that executive functions may influence performance in the GDT. In support of this hypothesis, there were no statistically significant differences between the two groups in WCST, and no differences in performance in the GDT. This current study outlined that performance of the GDT was also related to dealing with feedback with regard to winning and losing. The adverse selections of patients with eating disorders were obviously more than those of the control group, and safer decisions of patients were often less in response to positive feedback [48]. Similar results were shown in patients with attention deficit hyperactivity disorder (ADHD) [27]. Therefore, performance deficits in the GDT were hypothesised to be related to disadvantageous utilisation of feedback. In this current study, no significant difference was found in dealing with feedback or in other performance measures for the GDT between two groups.

Although alexithymia individuals may have impairment of feedback processing in the IGT, the ability of using feedback implicated in the GDT remained intact. Research has shown that this dissociation can been explained in that the two decision making tests depend on the two processes to a certain extent $[49,50]$. Several studies have indicated that the difference between GDT and IGT is similar to a shift from explicit to implicit knowledge [47, 51]. In addition, while executive functions are not as important as the use of feedback for exploring some rules in the IGT, they are particularly important for the use of favorable strategies in the GDT [52]. Thus, future studies will need to investigate the dissociation between decision-making under ambiguity and risk using the methods such as electrophysiology and neuroimaging.

There are some limitations of this study. First, as the findings of several studies involving both general and clinical populations have suggested, alexithymia is a stable personality trait $[53,54]$. While studies of alexithymia involving general populations may offer more information reflecting alexithymic trait, patient studies can focus specifically on the association between alexithymia and susceptibility to psychiatric symptoms. Second, our final sample included participants who ranged from 20 to 21 years old. It is well documented that age has an influence on personality, although the development of personality is relatively mature and stable after 18 years of age. Further investigations are required to determine the role of alexithymia among different age groups in general population and in psychosomatic and psychiatric disorders.

\section{Conclusion}

Our current study shows that alexithymia selectively influences decision-making under ambiguity. Our 
findings support the hypothesis that emotions may modulate human behaviour and cognition, especially decision making. In addition, we sought to reveal how emotions affect decision making under different conditions and other cognitive functions. Importantly, the findings extend the view that personality traits have impact on decision making. Further studies may utilise clinical populations to clarify the results and neural mechanisms through the method of functional magnetic resonance imaging and event-related potentials.

\section{Abbreviations}

ACC: Anterior cingulate cortex; ADHD: Attention deficit hyperactivity disorder; dIPFC: Dorsolateral prefrontal cortex; GDT: Game of dice task; IGT: lowa Gambling task; OFC: Orbitofrontal prefrontal cortex; SAS: Self-rating anxiety scale; SCRs: Skin conductance responses; SDS: Self-rating depression scale; SMH: Somatic-marker hypothesis; TAS-20: Toronto alexithymia scale; VMPFC: Ventromedial prefrontal cortex; WCST: Wisconsin card sorting test

\section{Acknowledgements}

We thank all the participants in this study. All authors also acknowledge the valuable support from Long Zhang, Fangfang Chen and Yaoting Sun.

\section{Funding}

This research was supported by the Natural Science Foundation of China $(81,301,176,31571149,91,432,301)$, Young Talents Foundation of Anhui Province in China (gxyqZD2016042). The funding sources had no role in study design, data collection and analysis, or preparation of the manuscript.

\section{Availability of data and materials}

Data in the study will not be shared. It was not in accordance with participants' written informed consent.

\section{Authors' contributions}

LZ was primarily responsible for the design of the study, the analysis of the data and writing the manuscript. XW, YZ, HCL contributed to subjects recruitment and data collection. FQY and CYZ played a role in conducting statistical analyses. The research co-ordinator of our project was WK. He was responsible for design of the study and writing the protocol. All authors have revised and approved the final manuscript.

\section{Ethics approval and consent to participate}

Our study was approved by the Ethics Committee at Anhui Medica University, China. Written informed consents were obtained from all the participants before the study.

\section{Consent for publication}

Not applicable.

\section{Competing interests}

The authors declare that they have no competing interests.

\section{Publisher's Note}

Springer Nature remains neutral with regard to jurisdictional claims in published maps and institutional affiliations.

\section{Author details}

'Department of Medical Psychology, Chaohu Clinical Medical College, Anhui Medical University, Hefei, China. ${ }^{2}$ Department of Neurology, the First Affiliated Hospital of Anhui Medical University, Hefei, China. ${ }^{3}$ Collaborative Innovation Center of Neuropsychiatric Disorder and Mental Health, Hefei, Anhui Province, China. ${ }^{4}$ Anhui Province Key Laboratory of Cognition and Neuropsychiatric Disorders, Anhui Medical University, Hefei, China.
Received: 21 February 2017 Accepted: 10 November 2017

Published online: 28 November 2017

\section{References}

1. Ong DC, Zaki J, Goodman ND. Affective cognition: exploring lay theories of emotion. Cognition. 2015;143:141-62.

2. Lang PJ, Davis M. Emotion, motivation, and the brain: reflex foundations in animal and human research. Prog Brain Res. 2006; 156(0079-6123 (Print)):3-29.

3. Mathews A, MacLeod C. Cognitive vulnerability to emotional disorders. Annu Rev Clin Psychol. 2005;1:167-95.

4. Sifneos PE, Apfel-Savitz R, Frankel FH. The phenomenon of 'alexithymia'. Observations in neurotic and psychosomatic patients. Psychother Psychosom. 1977;28(1-4):47-57.

5. Taylor GJ, Bagby RM. Measurement of alexithymia. Recommendations for clinical practice and future research. Psychiatr Clin North Am. 1988;11(3): 351-66.

6. Zhang L, Zhu C, Ye R, Cao Z, Tian Y, Yang P, Hu P, Wang K. Impairment of conflict processing in alexithymic individuals. Neurosci Lett. 2011;504(3):261-4.

7. Aite A, Barrault S, Cassotti M, Borst G, Bonnaire C, Houdé O, Varescon I, Moutier S. The impact of alexithymia on pathological gamblers' decision making: a preliminary study of gamblers recruited in "sportsbook" casinos. Cogn Behav Neurol. 2014;27(2):59-67.

8. Brand M, Labudda K, Markowitsch HJ, Markowitsch HJ. Neuropsychological correlates of decision-making in ambiguous and risky situations. Neural Netw. 2006:19(8):1266-76.

9. Bechara A, Damasio AR, Damasio H, Anderson SW. Insensitivity to future consequences following damage to human prefrontal cortex. Cognition. 1994;50(1-3):7-15.

10. Brand M, Fujiwara E, Borsutzky S, Kalbe E, Kessler J, Markowitsch HJ. Decision-making deficits of korsakoff patients in a new gambling task with explicit rules: associations with executive functions. Neuropsychology. 2005; 19(3):267-77.

11. Hampson SE, Andrews JA, Barckley M, Lichtenstein E, Lee ME. Personality traits, perceived risk, and risk-reduction behaviors: a further study of smoking and radon. Health Psychol. 2006;25(4):530-6.

12. Suzuki A, Hirota A, Takasawa N, Shigemasu K. Application of the somatic marker hypothesis to individual differences in decision making. Biol Psychol. 2003:65(1):81-8

13. Bechara A, Damasio H, Tranel D, Damasio AR. The lowa gambling task and the somatic marker hypothesis: some questions and answers. Trends Cogn Sci. 2005;9(4):159-62. discussion 162-154

14. Hebscher M, Barkan-Abramski M, Goldsmith M, Aharon-Peretz J, Gilboa A Memory, decision-making, and the Ventromedial prefrontal cortex (vmPFC): the roles of Subcallosal and posterior Orbitofrontal cortices in monitoring and control processes. Cereb Cortex. 2015;

15. Donahue CA-O, Lee DA-OX. Dynamic routing of task-relevant signals for decision making in dorsolateral prefrontal cortex. Nat Neurosci. 2015;18(2):295-301.

16. Jollant F, Lawrence NS, Olie E, O'Daly O, Malafosse A, Courtet P, Phillips ML. Decreased activation of lateral orbitofrontal cortex during risky choices under uncertainty is associated with disadvantageous decision-making and suicidal behavior. Neurolmage. 2010;51(3):1275-81.

17. Pujara MS, Wolf RC, Baskaya MK, Koenigs M. Ventromedial prefrontal cortex damage alters relative risk tolerance for prospective gains and losses. Neuropsychologia. 2015;79(Pt A):70-5.

18. Brand M, Franke-Sievert C, Jacoby GE, Markowitsch HJ, Tuschen-Caffier B. Neuropsychological correlates of decision making in patients with bulimia nervosa. Neuropsychology. 2007;21(6):742-50.

19. Delazer M, Sinz H, Zamarian L, Benke T. Decision-making with explicit and stable rules in mild Alzheimer's disease. Neuropsychologia. 2007:45(8):1632-41.

20. Labudda K, Woermann FG, Mertens M, Pohlmann-Eden B, Markowitsch HJ, Brand M. Neural correlates of decision making with explicit information about probabilities and incentives in elderly healthy subjects. Exp Brain Res. 2008;187(4):641-50.

21. Sutherland MT, Carroll AJ, Salmeron BJ, Ross TJ, Stein EA. Insula's functional connectivity with ventromedial prefrontal cortex mediates the impact of trait alexithymia on state tobacco craving. Psychopharmacology. 2013; 228(1):143-55

22. van der Velde J, van Tol MJ, Goerlich-Dobre KS, Gromann PM, Swart M, de Haan L, Wiersma D, Bruggeman R, Krabbendam L, Aleman A. 
Dissociable morphometric profiles of the affective and cognitive dimensions of alexithymia. Cortex. 2014;54:190-9.

23. Feldmanhall $\mathrm{O}$, Dalgleish $\mathrm{T}$, Mobbs D. Alexithymia decreases altruism in real social decisions. Cortex. 2013:49(3):899-904.

24. Bechara A, Tranel D, Damasio H. Characterization of the decision-making deficit of patients with ventromedial prefrontal cortex lesions. Brain. 2000; 123(Pt 11):2189-202.

25. Ji GJ, Yu F, Liao W, Wang K. Dynamic aftereffects in supplementary motor network following inhibitory transcranial magnetic stimulation protocols. Neurolmage. 2017;149:285-94.

26. Boller JK, Barbe MT, Pauls KA, Reck C, Brand M, Maier F, Fink GR, Timmermann L, Kalbe E. Decision-making under risk is improved by both dopaminergic medication and subthalamic stimulation in Parkinson's disease. Exp Neurol. 2014;254:70-7.

27. Matthies S, Philipsen A, Svaldi J. Risky decision making in adults with ADHD. J Behav Ther Exp Psychiatry. 2012;43(3):938-46.

28. Tien AY, Spevack TV, Jones DW, Pearlson GD, Schlaepfer TE, Strauss ME. Computerized Wisconsin card sorting test: comparison with manual administration. Kaohsiung J Med Sci. 1996;12(8):479-85.

29. Zhu X, Yi J, Yao S, Ryder AG, Taylor GJ, Bagby RM. Cross-cultural validation of a Chinese translation of the 20-item Toronto Alexithymia scale. Compr Psychiatry. 2007:48(5):489-96.

30. Scarpazza C, di Pellegrino G, Ladavas E. Emotional modulation of touch in alexithymia. Emotion (Washington DC). 2014;14(3):602-10.

31. Zung WW. A self-rating depression scale. Arch Gen Psychiatry. 1965;12 63-70.

32. Zung WW. A rating instrument for anxiety disorders. Psychosomatics. 1971; 12(6):371-9.

33. Bechara A, Damasio H, Damasio AR, Lee GP. Different contributions of the human amygdala and ventromedial prefrontal cortex to decision-making. J. Neurosci. 1999;19(13):5473-81.

34. Bechara A. lowa gambling task professional manual. Lutz: Psychological Assessment Resources; 2008.

35. Poletti M, Frosini D, Pagni C, Lucetti C, Del Dotto P, Tognoni G, Ceravolo $R$ Bonuccelli U. Alexithymia may modulate decision making in patients with de novo Parkinson's disease. Funct Neurol. 2011;26(3):127-31.

36. Murphy FC, Rubinsztein JS, Michael A, Rogers RD, Robbins TW, Paykel ES, Sahakian BJ. Decision-making cognition in mania and depression. Psychol Med. 2001;31(4):679-93.

37. Schmitt WA, Brinkley CA, Newman JP. Testing Damasio's somatic marker hypothesis with psychopathic individuals: risk takers or risk averse? J Abnorm Psychol. 1999;108(3):538-43.

38. Honkalampi K, Koivumaa-Honkanen H, Tanskanen A, Hintikka J, Lehtonen J, Viinamaki H. Why do alexithymic features appear to be stable? A 12-month follow-up study of a general population. Psychother Psychosom. 2001;70(5): 247-53.

39. Luminet O, Bagby RM, Taylor GJ. An evaluation of the absolute and relative stability of alexithymia in patients with major depression. Psychother Psychosom. 2001;70(5):254-60.

40. Vermeulen NLO, Corneille O. Alexithymia and the automatic processing of affective information: evidence from the affective priming paradigm. Cognit Emot. 2006;20(1):64-91.

41. Mantani T, Okamoto Y, Shirao N, Okada G, Yamawaki S. Reduced activation of posterior cingulate cortex during imagery in subjects with high degrees of alexithymia: a functional magnetic resonance imaging study. Biol Psychiatry. 2005;57(9):982-90.

42. Zhang L, Wang K, Zhu C, Yu F, Chen X. Trait anxiety has effect on decision making under ambiguity but not decision making under risk. PLoS One. 2015;10(5):e0127189.

43. Zhang L, Dong Y, Ji Y, Tao R, Chen X, Ye J, Zhang L, Yu F, Zhu C, Wang K. Trait-related decision making impairment in obsessive-compulsive disorder: evidence from decision making under ambiguity but not decision making under risk. Sci Rep. 2015;5:17312.

44. Brand M, Heinze K, Labudda K, Markowitsch HJ. The role of strategies in deciding advantageously in ambiguous and risky situations. Cogn Process. 2008;9(3):159-73.

45. Brand M, Kalbe E, Kracht LW, Riebel U, Munch J, Kessler J, Markowitsch HJ. Organic and psychogenic factors leading to executive dysfunctions in a patient suffering from surgery of a colloid cyst of the foramen of Monro. Neurocase. 2004:10(6):420-5.
46. Labudda K, Frigge K, Horstmann S, Aengenendt J, Woermann FG, Ebner A Markowitsch HJ, Brand M. Decision making in patients with temporal lobe epilepsy. Neuropsychologia. 2009;47(1):50-8.

47. Starcke K, Tuschen-Caffier B, Markowitsch HJ, Brand M. Dissociation of decisions in ambiguous and risky situations in obsessive-compulsive disorder. Psychiatry Res. 2010;175(1-2):114-20.

48. Svaldi J, Brand M, Tuschen-Caffier B. Decision-making impairments in women with binge eating disorder. Appetite. 2010;54(1):84-92.

49. Brand M. Does the feedback from previous trials influence current decisions? A study on the role of feedback processing in making decisions under explicit risk conditions. J Neuropsychol. 2008;2(Pt 2):431-43.

50. Starcke K, Tuschen-Caffier B, Markowitsch HJ, Brand M. Skin conductance responses during decisions in ambiquous and risky situations in obsessivecompulsive disorder. Cognitive neuropsychiatry. 2009;14(3):199-216.

51. Cavedini P, Zorzi C, Baraldi C, Patrini S, Salomoni G, Bellodi L, Freire RC, Perna $\mathrm{G}$. The somatic marker affecting decisional processes in obsessivecompulsive disorder. Cognitive neuropsychiatry. 2012;17(2):177-90.

52. Weisman O, Zagoory-Sharon O, Schneiderman I, Gordon I, Feldman R. Plasma oxytocin distributions in a large cohort of women and men and their gender-specific associations with anxiety. Psychoneuroendocrinology. 2013;38(5):694-701.

53. Luminet $\mathrm{O}$, Rokbani $\mathrm{L}$, Ogez $\mathrm{D}$, Jadoulle $\mathrm{V}$. An evaluation of the absolute and relative stability of alexithymia in women with breast cancer. J Psychosom Res. 2007;62(6):641-8.

54. Tolmunen T, Heliste M, Lehto SM, Hintikka J, Honkalampi K, Kauhanen J. Stability of alexithymia in the general population: an 11-year follow-up. Compr Psychiatry. 2011;52(5):536-41.

\section{Submit your next manuscript to BioMed Central and we will help you at every step:}

- We accept pre-submission inquiries

- Our selector tool helps you to find the most relevant journal

- We provide round the clock customer support

- Convenient online submission

- Thorough peer review

- Inclusion in PubMed and all major indexing services

- Maximum visibility for your research

Submit your manuscript at www.biomedcentral.com/submit
C Biomed Central 